\title{
B Cell Production of Both OPG and RANKL is Significantly Increased in Aged Mice
}

\author{
Yan $\mathrm{Li}^{1,2}$, Masakazu Terauchi ${ }^{1}$, Tatyana Vikulina, ${ }^{1}$ Susanne Roser-Page, ${ }^{3}$ and \\ M. N. Weitzmann ${ }^{1,2,3, *}$
}

${ }^{I}$ Division of Endocrinology and Metabolism and Lipids, Department of Medicine, Emory University School of Medicine, Atlanta, GA, 30322, USA

${ }^{2}$ Emory Winship Cancer Institute, Emory University, Atlanta, GA, 30322, USA

${ }^{3}$ Atlanta Department of Veterans Affairs Medical Center, Decatur, Georgia 30033, USA

Current Address: Masakazu Terauchi: Department of Women's Health, Tokyo Medical and Dental University, Tokyo, Japan

Current Address: Yan Li: Emory Winship Cancer Institute, Emory University, 1365 Clifton Road NE, Suite B3012, Atlanta, GA 30322, USA

\begin{abstract}
Aging is a risk factor for osteoclastic bone loss and bone fracture. Receptor activator of NF- $\kappa \mathrm{B}$ ligand (RANKL) is the key effector cytokine for osteoclastogenesis and bone resorption, and is moderated by its decoy receptor osteoprotegerin (OPG). The development of an inflammatory environment during aging leads to increased bone resorption and loss of bone mineral density (BMD). Interestingly, animal and clinical studies show that OPG is actually increased in aging but fails to fully compensate for endogenous RANKL. Osteoblast- and B-lineage cells are significant sources of physiological OPG, however osteoblast OPG production declines with age, suggesting that elevated OPG in aging may be a consequence of changes in B cell function. In this study we examined BMD and indices of trabecular bone structure during aging, and B cell production of both RANKL and OPG in young and aged mice. Our data reveal significant loss of BMD and trabecular structure with age commensurate with significantly elevated concentrations of both OPG and RANKL in aged mice, and a decline in B cell populations in aged animals. Taken together our data suggest that B cells may be responsible for the elevated concentrations of OPG during aging and are essential to counteract excessive ageassociated bone resorption. Paradoxically, B cells themselves likely contribute RANKL in aging and the loss of B cells with age may further contribute to the imbalance in OPG relative to RANKL that predisposes age-associated bone loss.
\end{abstract}

Keywords: Aging, OPG, RANKL, B cells, Osteoporosis, Osteoclast.

\section{INTRODUCTION}

Aging is a pivotal risk factor for bone loss and for the development of osteoporosis [1] a disease of the skeleton associated with increased risk of bone fractures.

Components of the immune and skeletal systems are deeply integrated and as a consequence, changes in immune function are transduced through the immuno-skeletal interface, an association of common cells and cytokine effectors that mediates distinct albeit different functions in the immune and skeletal systems. Alterations in immune function thus lead to significant changes in skeletal metabolism, often causing imbalanced bone turnover with consequent loss of BMD. Prominent cellular components of

*Address correspondence to this author at the Division of Endocrinology \& Metabolism \& Lipids, Emory University School of Medicine, 101 Woodruff Circle, 1305 WMB, Georgia 30322-0001; Tel: 404-727-1389;

Fax: 404-727-1300; E-mail: mweitzm@emory.edu the immuno-skeletal interface include monocytes (the source of osteoclast precursors) and lymphocytes (both B cells and T cells).

Osteoclasts form in the bone marrow (BM) in the presence of permissive levels of Macrophage colony stimulating factor (M-CSF), and the key osteoclastogenic cytokine RANKL is moderated by its physiological decoy receptor OPG [2]. In conditions of physiological bone remodeling bone-forming osteoblasts have historically been considered to be an important source of OPG in the bone microenvironment. Interestingly, human B cells have also been recognized as a significant source of OPG [3] although the significance of this discovery for bone biology was not immediately appreciated. We recently reported that B cell deficiency in mice leads to elevated basal osteoclastic bone resorption and diminished BMD and volume, as a consequence of a bone marrow deficiency in OPG. Rescue studies involving transplantation of B cells back into B cell knock out (KO) mice restored OPG to wild type (WT) levels 
and prevented the development of osteoporosis [4]. Using WT mice to quantify B-lineage sources of OPG production we further demonstrated that mature B cells accounted for $45 \%$ of total bone marrow OPG production. In addition, B cell precursors, immature B cells and plasma cells further contributed OPG to the bone microenvironment with the entire B cell linage accounting for up to $64 \%$ of total OPG production [4]. Interestingly, as previously reported for human B cells in vitro [3] CD40 costimulation by T cells led to enhanced $\mathrm{B}$ cell OPG production in mice in vivo, further defining an important role for $\mathrm{T}$ cell-mediated regulation of $\mathrm{B}$ cell OPG. Indeed bone marrow OPG production was significantly compromised by $\mathrm{T}$ cell deficiency and/or by genetic ablation of $\mathrm{CD} 40$ and $\mathrm{CD} 40 \mathrm{~L}$ costimulatory molecules leading to elevated bone resorption and reduced bone mass in each of these models [4]. Taken together these data demonstrated that $\mathrm{B}$ cell and $\mathrm{T}$ cell function is important for maintenance of basal bone homeostasis and the RANK/RANKL/OPG axis is thus a key mechanism by which immune cells regulate or disrupt physiological bone turnover [5-8]. Indeed, we have reported that a decline in B cell expression of OPG and a concomitant increase in B cell RANKL, drives bone loss in a rat model of HIV-infection [9], a malady long associated with osteoporosis in humans [6]. In a recent clinical study we further demonstrated the existence of this B cell RANKL/OPG imbalance in the context of human HIV-infection, where the $\mathrm{B}$ cell RANKL/OPG ratio was significantly correlated with loss of BMD [10].

Numerous studies show that OPG production is also imbalanced in aged humans and rodents, and although osteoblast-specific production of OPG is typically reported to be diminished [11-13] surprisingly, total OPG production is routinely found to be elevated with age in both men and women [11-17]. The source of elevated OPG in aging remains unknown.

It is well established that aging leads to immunosenescence, a progressive decline in immune responsiveness to exogenous antigens and a paradoxical increase in autoimmunity [18]. Immunosenescence strongly impacts B cell linages and may be a reflection of intrinsic B cell defects as well as altered microenvironment, including helper $\mathrm{T}$ cell function [18]. Aging further promotes accumulation of autoreactive $\mathrm{T}$ and $\mathrm{B}$ cells $[19,20]$ leading to a chronic inflammatory state that has been referred to as "Inflammaging" [21]. These disruptions in B cell function brought about by aging thus have the capacity to influence the immuno-skeletal interface impacting RANKL and OPG production and hence affecting the process of osteoclastic bone resorption.

In this study we examined changes in BMD and bone structure with aging in mice and quantified total serum RANKL and OPG, and bone marrow B cell RANKL and OPG production. We report that $\mathrm{B}$ cell OPG production significantly increase with aging, although B cells themselves contribute significant concentrations of RANKL in aged mice.

\section{METHODS}

All reagents were purchased from the Sigma-Aldrich Chemical Co. (St. Louis, MO), unless otherwise indicated.

\subsection{Mice}

All animal studies were approved by the Emory University Animal Care and Use Committee and were conducted in accordance with the NIH Laboratory Guide for the Care and Use of Laboratory Animals.

Mice were housed under specific pathogen free conditions and were fed gamma-irradiated 5V02 mouse chow (Purina Mills, St. Louis, MO), and autoclaved water $a d$ libitum. The animal facility was kept at $23 \pm 1{ }^{\circ} \mathrm{C}$, with $50 \%$ relative humidity and a 12/12 light/dark cycle.

Young (6-12 weeks of age) female C57BL6 WT were from Jackson Labs (Bar Harbor, ME) and aged (20-24 month old) mice were from the National Institute on Aging (NIA) aged mouse colony at Charles River Laboratories (Wilmington, MA).

\subsection{Bone Densitometry}

BMD $\left(\mathrm{g} / \mathrm{cm}^{2}\right)$ quantifications were performed in anesthetized mice by Dual energy X-ray absorptiometry (DXA) using a PIXImus 2 bone densitometer (GE Medical Systems) and calibrated daily using a factory-supplied phantom. Anesthetized mice were placed on the imaging tray in a prostrate position. Total body DXA was performed and region of interest boxes placed to quantify anatomical sites including lumbar spine and femur. The left and right femurs were averaged for each mouse and the mean used for group calculations. Cross sectional BMD measurements at specific ages including 2, 3, 4, 6, 10, 15 and 21 months were repurposed from baseline BMDs from multiple studies previously conducted at these time points. The short-term in vitro reproducibility is $1.7 \%$ [22].

\subsection{Micro-Computed Tomography}

Micro-Computed Tomography $(\mu \mathrm{CT})$ was performed in L3 vertebrae ex vivo to assess trabecular bone microarchitecture using a $\mu \mathrm{CT} 40$ scanner (Scanco Medical AG, Bruettisellen, Switzerland) calibrated weekly with a factory-supplied phantom. A total of 500 tomographic slices at a voxel size of $6 \mu \mathrm{m}(70 \mathrm{kVp}$ and $114 \mathrm{~mA}$, and $200 \mathrm{~ms}$ integration time) were taken at the L3 vertebra (total area of $3.0 \mathrm{~mm}$ ) and trabecular bone segmented from the cortical shell. Projection images were reconstructed using the autocontour function for trabecular bone. Representative vertebral samples based on mean BV/TV were reconstructed in $3 \mathrm{D}$ to generate visual representations. Indices and units were standardized per published guidelines [23].

\subsection{Biochemical Indices of Bone Resorption and Bone Active Cytokines}

CTx was quantified in mice serum using RATlaps (CTx) ELISAs (Immunodiagnostic Systems Inc. Fountain Hills AZ). OPG or RANKL was quantified in serum using mouse 
specific ELISAs (MOP00 for mouse OPG and MTR00 for RANKL) R \& D Systems, Minneapolis, MN.

\subsection{Cell purification, and RANKL and OPG ELISAs}

Bone marrow was extracted from long bones by insertion into a $0.5 \mathrm{ml}$ centrifuge tube with a hole pierced into the bottom with an 18 gauge needle, and the tube inserted into a $1.5 \mathrm{ml}$ centrifuge tube and spun at $13,500 \mathrm{x} \mathrm{g}$ for 60 seconds. Splenocytes were recovered from spleens following disaggregation by gentle scraping with glass slides. Recovered bone marrow and splenocytes were gently resuspended in 15 volumes of red cell lysis buffer $(1 \mathrm{mM}$ EDTA; $100 \mathrm{mM} \mathrm{KHCO} 3,1.7 \mathrm{M} \mathrm{NH}_{4} \mathrm{Cl}$; pH 7.3) for 10 minutes at room temperature followed by 2 washes with PBS. B cells were purified from bone marrow or spleen using immunomagnetic beads (Miltenyi Biotech, Auburn, CA).

Conditioned media was generated by plating $1 \times 10^{7}$ purified B lineage cells into 48 well plates in $1 \mathrm{ml} /$ well of RPMI 1640 supplemented with 10\% FBS and antibiotics. After $48 \mathrm{~h}$ media was collected for analysis of OPG or RANKL by mouse specific ELISAs (MOP00 for mouse OPG and MTR00 for RANKL) from R \& D Systems.

\subsection{Flow Cytometry}

To quantify B cell populations in spleen and bone marrow single cell suspensions $\left(10^{6}\right.$ cells/reaction $)$ were prepared and surface stained in FACS buffer (phosphatebuffered saline (PBS) containing 1\% Fetal Bovine Serum (FBS) for 30 minutes at $4^{\circ} \mathrm{C}$, and washed once in FACS buffer. B cells were stained using CD45R (B220)-APC or IgD-FITC (eBioscience Inc. (San Diego, CA) and gates established using isotype and fluorochrome-matched controls. Samples were acquired on an Accuri flow cytometer (BD Immunocytometry Systems).

\subsection{Statistical Analysis}

Statistical significance was determined using GraphPad InStat version 3.0 for Windows (GraphPad Software Inc. La Jolla, CA). Gaussian distribution was assessed using the Kolmogorov and Smirnov test. Simple comparisons were made using unpaired 2-tailed Students $t$ test or MannWhitney for non-parametric data. Multiple comparisons were performed using One-way ANOVA with Tukey's Multiple Comparison post hoc test. $\mathrm{P} \leq 0.05$ was considered statistically significant. Data are presented as Mean \pm SD unless otherwise indicated.

\section{RESULTS}

In mice, bone modeling proceeds until peak BMD occurs at around 5 months, at which point bone remodeling begins [24]. The skeleton is further impacted by reproductive senescence at $12-15$ months of age with a decline in estradiol production in females [25]. Age-associated immunosenescence begins at around 14 months of age [26] and mice are considered aged by around 24 months of age. Mice thus represent a time-compressed model of human skeletal, immunological and age-associated changes making them ideal for skeletal studies.

\subsection{Bone Densitometry}

BMD was quantified by DXA in mice at multiple ages including 2, 3, 4, 6, 10, 15 and 21 months. Our data (Fig. 1A) shows a gain in femoral BMD between 2 months of age and 10 months of age followed by a rapid decline in BMD between 10 and 15 , and 15 and 21 months of age and declining to the original 2-month baseline by 21 months. By contrast BMD peaked by 4 months at the lumbar spine and then declined progressively thereafter falling below the 2month baseline by 21 months. By contrast total body measurements were more stable showing a peak between 10 and 15 months with a slow decline after 10 months of age.

\subsection{Micro-Computed Tomography}

Because DXA underestimates the trabecular compartment we further performed high-resolution $(6 \mu \mathrm{m})$ $\mu \mathrm{CT}$ of isolated vertebral bodies (a trabecular rich region) from mice just prior to immunological and reproductive senescence (12 months of age) and in aged mice (24 months of age). Representative 3D visual reconstructions are shown in Fig. (1B). Quantitative microarchitectural indices of trabecular bone structure were computed for multiple mice (Table 1) and reveal that vertebral trabecular bone volume fraction (BV/TV) was significantly decreased between the ages of 12 and 24 months. Interestingly, the decline in $\mathrm{BV} / \mathrm{TV}$ was predominantly a consequence of an increase in tissue volume (TV) a reflection of total bone size with only a modest decline in bone volume (BV) that failed to reach statistical significance. Although the data suggest a continued increase in total bone size over the life of the mouse, overall BMD reflected by TV.D was significantly diminished in the aged mice suggesting an overall loss of bone mass. Diminished trabecular structure was reflected by a significant increase in trabecular separation (Tb. Sp.) a reflection of the distance between trabeculae, while trabecular thickness (Tb. Th.) and trabecular number ( $\mathrm{Tb}$. N.) were significantly decreased between 12 and 24 months.

Taken together these data reveal a significant decline in trabecular BMD and volume with deteriorating structural indices following immunological senescence in mice.

\subsection{Biochemical Indices of Bone Resorption}

C-terminal Telopeptide of type I collagen (CTx), a specific and sensitive biochemical index of global bone resorption was quantified in serum of 12 and 24 month of mice and revealed a significance increase in bone resorption at 24 months (Fig. 1C).

\subsection{Global Serum Levels of OPG and RANKL are Elevated During Aging in Mice}

Several studies in humans have reported that serum OPG production increases with age in both men and women [1117]. To validate the mouse as a model for aging studies we quantified OPG and RANKL in the serum of 3,12 and 24 


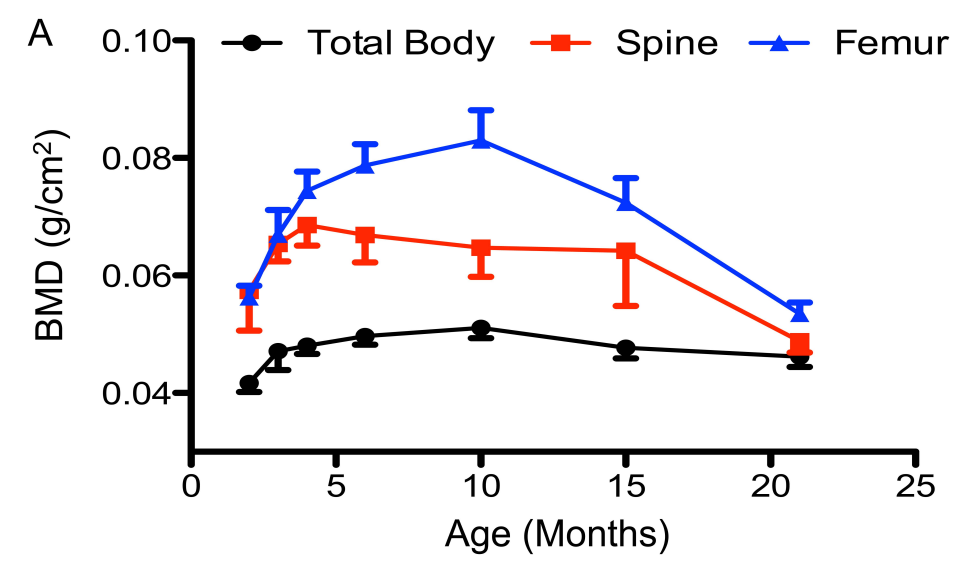

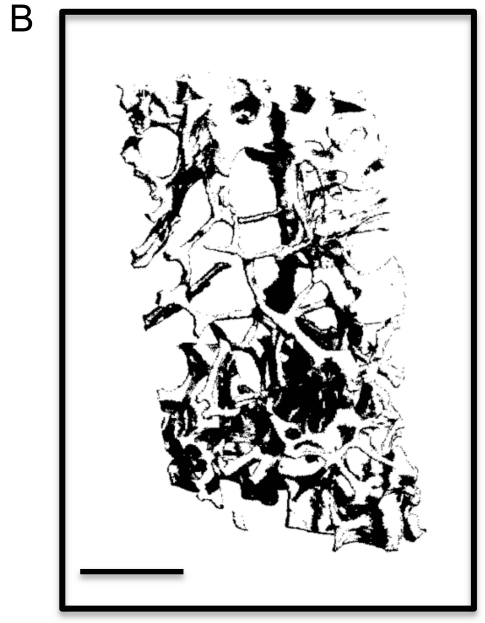

12 Months

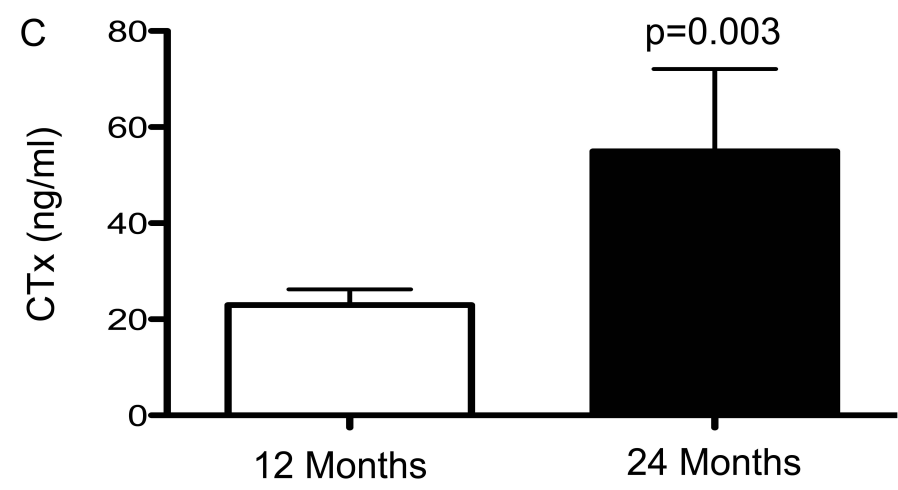

Fig. (1). Changes in BMD, trabecular bone structure and bone resorption in aged mice. (A) BMD was quantitated in female C57BL6 mice at 2, 3, 4, 6, 10, 15 and 21 months of age by DXA. Data presented for Lumbar Spine (Red), average of left and right femur for each mouse (Blue) and for total body (Black). $\mathrm{N}=6-21 \mathrm{mice} /$ data point. (B) Representative 3D $\mu \mathrm{CT}$ reconstructions for L3 vertebrae from 12 and 24 month old mice. Black scale bar $=500 \mu \mathrm{m}$. (C) Biochemical index (CTx) of in vivo bone resorption in 12 and 24 month old mice. $\mathrm{N}=18$ mice 12 months old and 5 mice 24 month old mice; Mann-Whitney test.

Table 1. $\mu \mathrm{CT}$ trabecular indices for lumbar spine in 12 and 24 month old mice.

\begin{tabular}{|c|c|c|c|c|}
\hline Trabecular Indices & 12 Month & 24 Month & $\%$ Change & $\mathbf{P}$ \\
\hline $\mathrm{TV}\left[\mathrm{mm}^{3}\right]:$ & $3.24 \pm 0.089$ & $3.87 \pm 0.30$ & +19.5 & $<0.0001$ \\
\hline
\end{tabular}


Table 1. contd....

\begin{tabular}{|c|c|c|c|c|}
\hline Trabecular Indices & 12 month & 24 month & $\%$ Change & $\mathbf{P}$ \\
\hline $\mathrm{BV} / \mathrm{TV}[\%]:$ & $0.1191 \pm 0.0247$ & $0.0892 \pm 0.0251$ & -25.1 & 0.0262 \\
\hline $\mathrm{Tb} . \mathrm{N}[/ \mathrm{mm}]$ : & $2.81 \pm 0.30$ & $2.14 \pm 0.38$ & -24.0 & 0.0011 \\
\hline Tb. Sp [mm]: & $0.3596 \pm 0.0386$ & $0.4869 \pm 0.0841$ & +35.4 & 0.0014 \\
\hline
\end{tabular}

Trabecular indices: tissue volume (TV), trabecular bone volume (BV), trabecular thickness (Tb. Th), trabecular seperation (Tb. Sp), trabecular number (Tb. N), trabecular total volumetric BMD (TV. D). Mean \pm SD of 8 (12 month old) and 9 (24 month old) mice per group. P values from Student's t test.
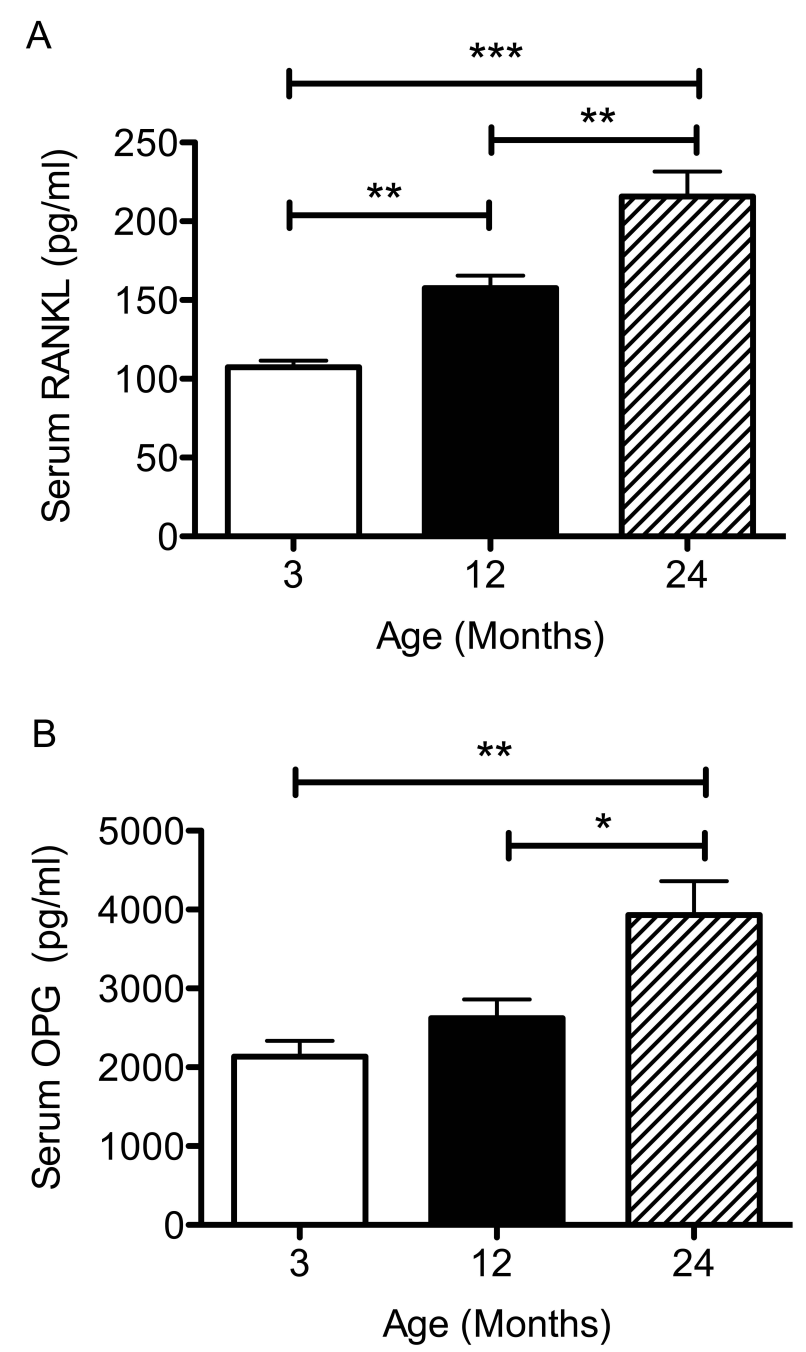

Fig. (2). Changes in serum OPG and RANKL production in young, mature and aged mice. The concentrations of circulating (A) RANKL and (B) OPG, were quantified in the serum of young (3 months), skeletally mature (12 months) and aged (24 month) female C57BL6 mice by ELISA. Data presented as mean \pm SEM, N=7-14 mice/group; ${ }^{*} \mathrm{p} \leq 0.05 ; * * \mathrm{p} \leq 0.01, * * * \mathrm{p} \leq 0.001$ by One-way ANOVA with Tukey's Multiple Comparison Test.

month old female mice and documented significant increase in both RANKL (Fig. 2A) and OPG (Fig. 2B).

\subsection{B Cell OPG and RANKL Production During Aging}

The role of B cells in the bone loss associated with aging in mice and humans is presently unclear. As B cells are a significant source of basal OPG, changes in B cell number and/or function, during aging, have the potential to impact osteoclastogenesis and bone resorption. We thus investigated whether aging affects $B$ cell OPG and/or RANKL production. Bone marrow and spleens were harvested from young (2 months old) and mice approaching old age (21 months old) and B cells purified by immunomagnetic 


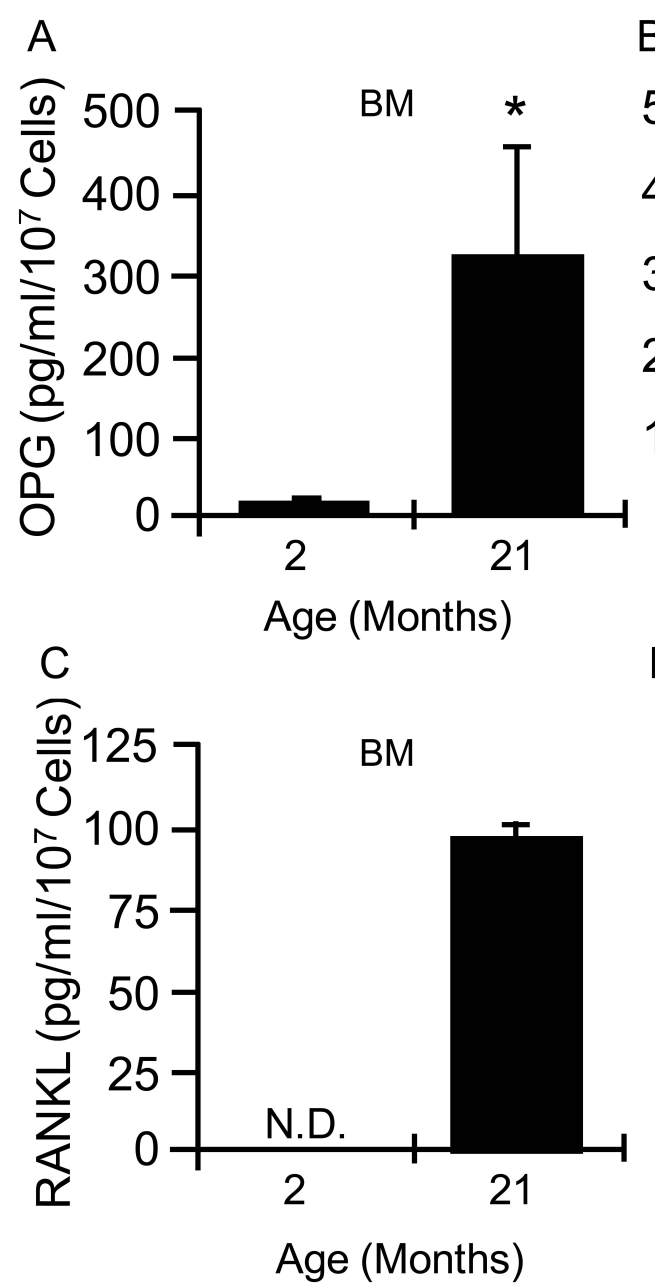

B

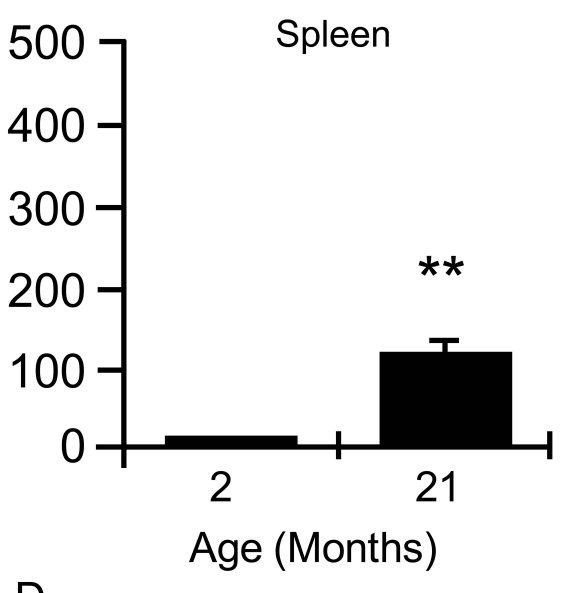

D

Age (Months)

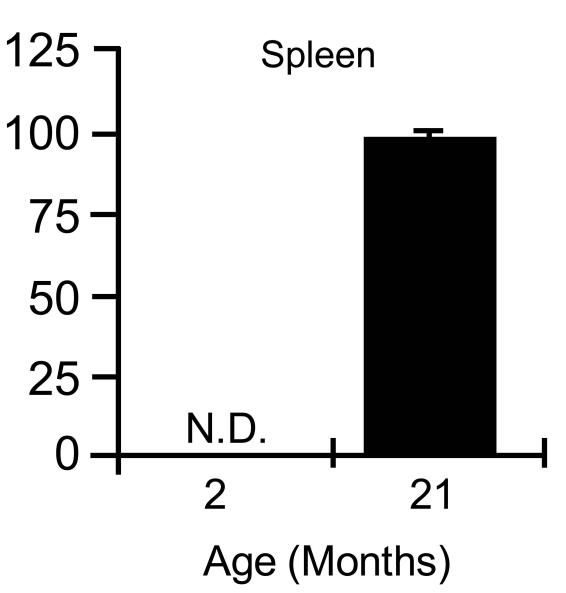

Fig. (3). Changes in OPG and RANKL production by B cells in young and aged mice. Production of OPG by B cells purified from (A) bone marrow (BM) and (B) spleen in young and old female C57BL6 mice. Production of RANKL by B cells purified from (C) bone marrow $(\mathrm{BM})$ and (D) spleen in young and old mice. B cells were immunomagnetically purified from young ( 2 months old) and old (21 month old) mice. OPG and RANKL production from $1 \times 10^{7}$ cells was quantitated in $48 \mathrm{~h}$ conditioned mediums by ELISA. N=6 mice/group; * $\mathrm{p} \leq 0.05$; $* * \mathrm{p} \leq 0.01$, Students t test.

selection. B cell conditioned mediums were generated and OPG and RANKL production by B cells was quantified $48 \mathrm{~h}$ later by commercial ELISAs. Our data reveal that B cells isolated from the bone marrow of aged mice secrete $\sim 25$ fold more OPG than an equivalent number of young B cells (Fig. 3A). OPG production by splenic B cells from old mice (Fig. 3B) was similarly elevated although by a smaller magnitude ( 12 fold). Interestingly, while no RANKL production was detectable by $\mathrm{B}$ cells isolated from the bone marrow or spleen of young mice, B cells from aged mice produced significant levels (Fig. 3C and D).

\subsection{B Cell Numbers are Diminished in Aging Mice}

In order to determine whether the C57BL6 mouse model used for our bone studies recapitulate the diminution of $\mathrm{B}$ cells associated with aging in humans [27] we quantified the B cell compartments of young mice ( 2 months of age) and old mice (21 months of age). Flow cytometric analysis of spleens and bone marrow from young and old mice did indeed reveal changes in B cell number. The spleens of aged mice contained $26 \%$ fewer B cells than in young mice (Fig. 4A). Similarly, $\mathrm{B} 220^{+}$cells in the bone marrow were decreased by $46 \%$ (Fig. 4B). The B220 marker represents mature B cells (B220 Bright $)$ as well as immature B cells and B cell precursor populations $\left(\mathrm{B} 220^{\mathrm{Dim}}\right)$ showing up as 2 peaks (Fig. 4B). To further determine changes specifically in mature B cells we further stained bone marrow with IgD a marker of mature B cells, which revealed that mature B cells were diminished by $49 \%$ (Fig. 4C).

\section{DISCUSSION}

Bone loss ensues during aging as a consequence of enhanced osteoclastic bone resorption relative to bone formation. Previous studies have reported that peak bone strength is achieved by 5 months of age in mice [24]. Our studies show that shortly after attainment of peak BMD the mouse skeleton begins to lose bone mass, although the 


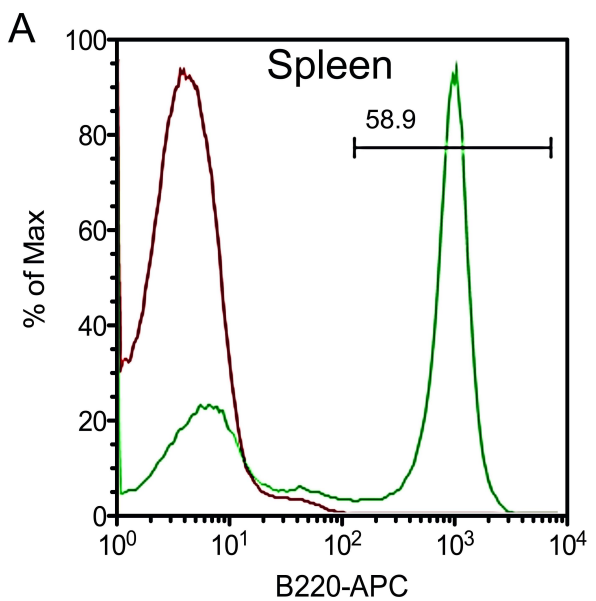

2 Months
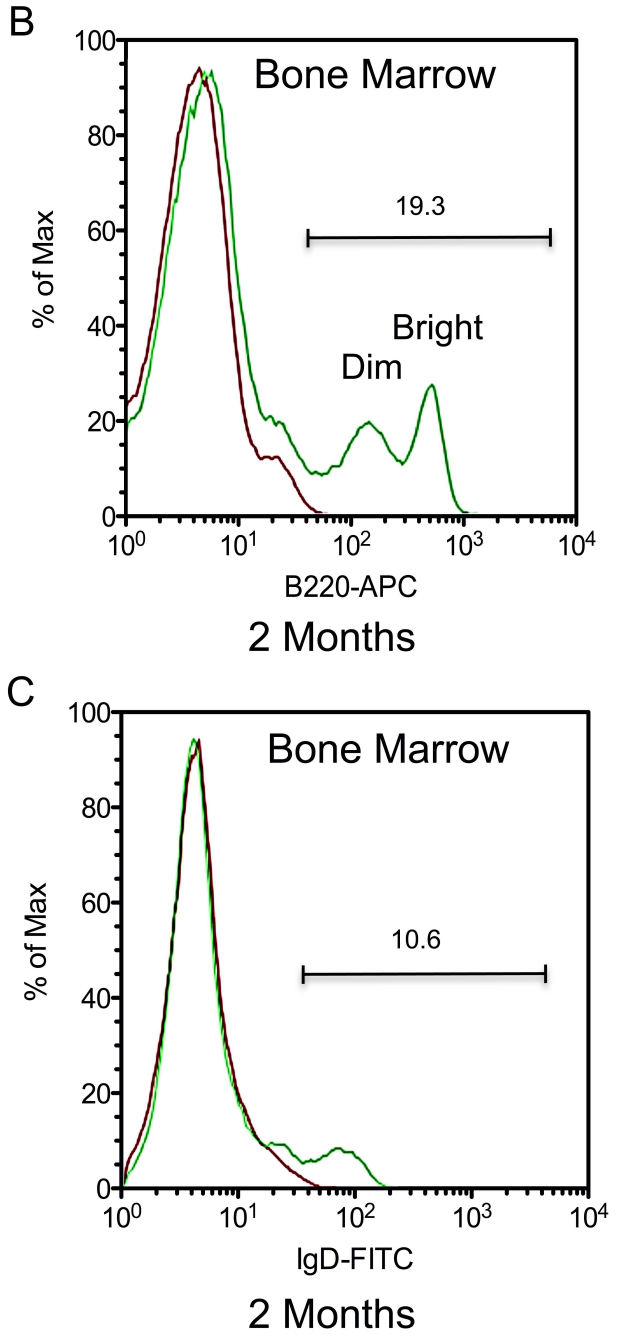

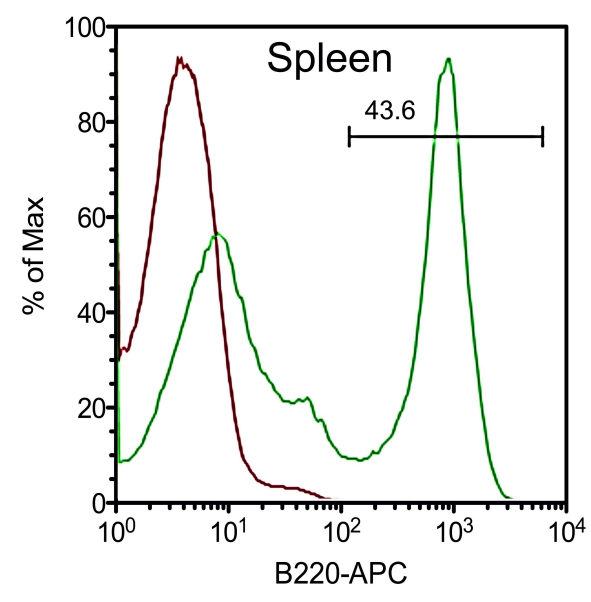

21 Months
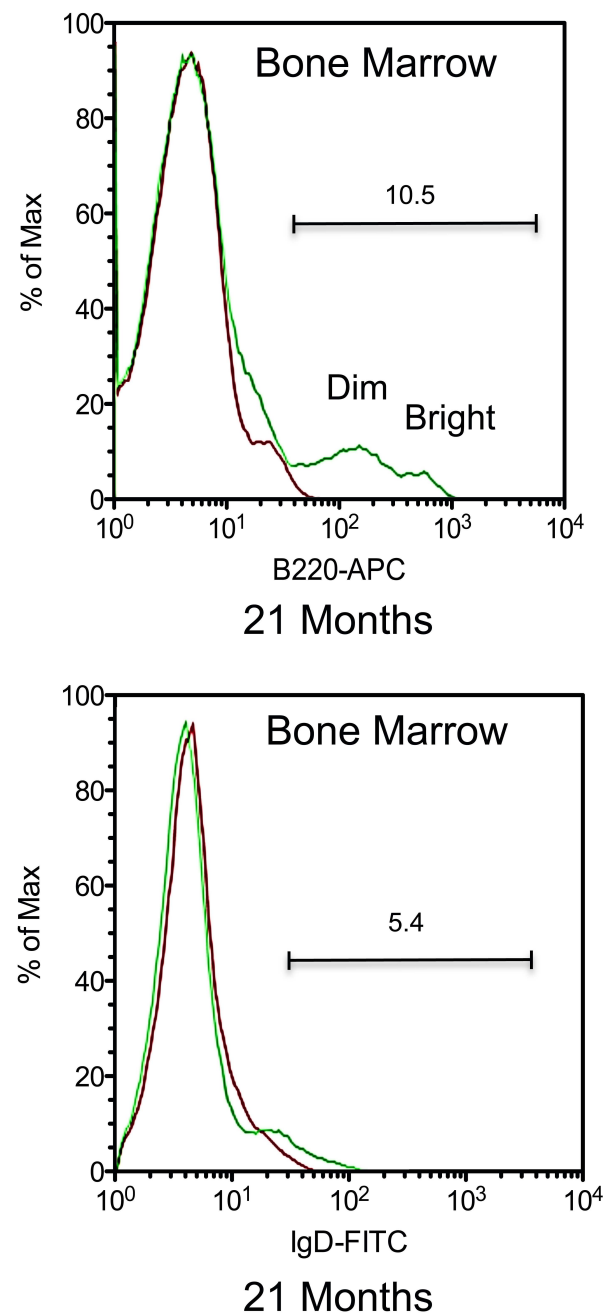

Fig. (4). B lineage cells are diminished in aged mice. Percentage of $B 220^{+}$cells (total B lineage) in the (A) Spleen and (B) bone marrow (BM) of young (2 months of age) and old (21 months of age) female C57BL6 mice. (C) Mature (IgD $\left.{ }^{+}\right)$B cells in the BM of 2 and 21 month old mice. Cells quantitated by Flow Cytometry. Isotype control shown in red.

temporal effect was somewhat anatomical site specific with lumbar spine degeneration beginning shortly after peak BMD (around 4 months of age) while femoral BMD peaked later at around 10 months before declining. Interestingly total body BMD was observed to be relatively stable, peaking between 10 and 15 months of age, followed by a slow modest downward trend between 10 and 20 months. Because DXA grossly underestimates trabecular bone, the 
most metabolically active compartment, the apparent stability of total body BMD is likely a reflection of cortical bone, being lost more slowly. Trabecular bone, more predominant in the spine, is rapidly lost in mice likely accounting for the early decline in total BMD observed in the spine, while cortical-rich femoral bone is observed to be maintained for a longer period of time. $\mu \mathrm{CT}$ analysis of the trabecular compartment of the lumbar spine reveled a significant decline in $\mathrm{BV} / \mathrm{TV}$, but interestingly this was largely a consequence of increased bone size (TV) likely due to ongoing periosteal bone apposition concurrent with endosteal bone resorption, leading to bone growth without significant trabecular bone gain and a net decline in trabecular BV/TV.

Reproductive senescence occurs between 12 and 15 months with a significant decline in estradiol production in female mice [25]. Immunosenescence in mice begins by 14 months [26] and both reproductive and immunosenescence may be key stages at which cortical bone loss begins and $\mathrm{B}$ cell RANKL and OPG production begin to change, although a detailed temporal analysis of OPG and RANKL production by $\mathrm{B}$ cells remains to be performed.

Surprisingly, animal and clinical studies suggest that OPG is increased with aging in men and women, despite elevated bone resorption. It has further been suggested that this rise in OPG may be a homeostatic mechanism to limit bone loss [14]. Interestingly, osteoblast derived OPG appears to decline with age and the source of elevated OPG in aging has remained unclear. Our data suggest that B cells are a likely source of these elevated OPG concentrations associated with aging.

Interestingly, B cells were not only associated with elevated concentrations of OPG, but also appear to be a significant source of RANKL in aged mice. The lack of RANKL expression in young animals is consistent with our previous study in which no difference in basal RANKL expression was observed between $\mathrm{B}$ cell replete and $\mathrm{B}$ cell deficient animals [4]. These data are further consistent with a recent study showing that conditional RANKL ablation in B cells fails to impact bone mass in estrogen-replete mice up to 7 months of age [28]. B cells have however been observed to secrete RANKL and may directly contribute to bone loss when activated as occurs in several pathological conditions including estrogen deficiency [28, 29], periodontal infection $[30,31]$, psoriatic arthritis [32] and HIV infection $[9,10]$.

Although aged B cells produced both OPG and RANKL, OPG production in the bone marrow outpaced that of RANKL by $\sim 3$ fold while spleen RANKL and OPG production was similar. Although the stoichiometry of the RANKL/OPG interaction in vivo is difficult to define, it is likely that an increase in RANKL would require more than an equivalent increase in OPG to fully compensate. Given that bone loss ensues in aging despite increased OPG (in mice and humans) the overall total increase in OPG from all sources is likely outpaced by the overall total increase in RANKL from all sources.

It should further be pointed out that we quantified soluble RANKL production by B cells only. While osteoblasts are associated predominantly with membrane bound RANKL, B cells are considered to produce mostly secreted RANKL, however, there may also be additional membrane associated RANKL that causes an increase in total RANKL exacerbating bone resorption. Furthermore, OPG is known to have other ligands beside RANKL, including TRAIL. Although we did not quantify TRAIL in our studies, it is possible that TRAIL may decoy some OPG. Thus membrane bound forms of RANKL and other OPG ligands may conspire to diminish the overall concentration of OPG available to neutralize RANKL.

Furthermore, TNF $\alpha$ has long been recognized as a key protagonist of bone loss in inflammatory conditions including rheumatoid arthritis [33], postmenopausal osteoporosis [7] and indeed in the context of aging [34]. TNF $\alpha$ can potently synergize with RANKL [35] at the level of RANK signal transduction [36] to further increase its osteoclastogenic potency and to activate bone resorption [37]. The double onslaught of increased bioavailable RANKL (free RANKL) and TNF $\alpha$ may push up resorption to levels that cannot be compensated for by the increases in OPG.

Our data suggest that aging leads to an upregulation in OPG by $B$ cells that compensates, in part, for elevated RANKL production, paradoxically also in part from B cells, thus partially mitigating age-associated bone resorption.

In adults, B lymphocytes are derived from hemopoietic stem cells (HSC) in the bone marrow that progress through a series of distinct developmental stages defined by various phenotypic markers and the rearrangement and expression of immunoglobulin genes [38]. Age-related defects in the proliferative potential of Common Lymphoid Progenitors (CLP) and their progeny, the pre-pro-B cells suggest that the ability of CLP to differentiate into pre-pro-B cells is compromised by immunosenescence [38]. It is generally agreed that B cell precursors are diminished with age as are memory B cells in the periphery [27, 39]. Clinical studies further report that both the frequency of circulating memory $\mathrm{B}$ cells and the absolute number are reduced in the elderly $[40,41]$. Although it has been suggested that homeostatic expansion of antigen-experienced memory B cells may continue to compensate and fill available niches [42], it is also now becoming apparent that available niches for B cells in germinal centers and lymph nodes also declines with age, leading to diminished space for B cell engraftment [43]. Our data in aged mice confirm this reduction on $\mathrm{B}$ cell populations in the spleen, which shows a modest decrease, as well as a large decline in bone marrow mature B cells and B cell precursor populations. This decline in B cells may in part, limit the capacity of this population to provide adequate OPG to offset enhanced RANKL-mediated bone resorption. By contrast, the decline in B cells may also limit the production of pro-resorptive RANKL produced.

In conclusion, our data suggest that age-associated alterations in B cell number and function lead to elevated B cell OPG production that likely protects against bone loss and limits its severity by compensating, in part, for elevated total and B cell RANKL production during aging. Our data 
suggest that B cells may represent a key target of future antiosteoporotic therapies to ameliorate senile osteoporosis.

\section{CONFLICT OF INTEREST}

The authors confirm that this article content has no conflict of interest.

\section{ACKNOWLEDGEMENTS}

MNW is supported, in part, by a grant from the Biomedical Laboratory Research \& Development Service of the VA Office of Research and Development (5I01BX000105) and by NIAMS grants (AR053607, AR056090 and AR059364) and NIA grant AG040013. The contents of this manuscript do not represent the views of the Department of Veterans Affairs, the National Institutes of Health or the United States Government.

\section{REFERENCES}

[1] Streckfus CF, Parsell DE, Streckfus JE, Pennington W, Johnson RB. Relationship between oral alveolar bone loss and aging among African-American and Caucasian individuals. Gerontology 1999; 45: 110-4.

[2] Teitelbaum SL. Bone resorption by osteoclasts. Science 2000; 289 : 1504-8.

[3] Yun TJ, Chaudhary PM, Shu GL, et al. OPG/FDCR-1, a TNF receptor family member, is expressed in lymphoid cells and is upregulated by ligating CD40. J Immunol 1998; 161: 6113-21.

[4] Li Y, Toraldo G, Li A, et al. B cells and T cells are critical for the preservation of bone homeostasis and attainment of peak bone mass in vivo. Blood 2007; 109: 3839-48.

[5] Ofotokun I, McIntosh E, Weitzmann MN. HIV: Inflammation and Bone. Curr HIV/AIDS Rep 2012; 9: 16-25.

[6] Ofotokun I, Weitzmann MN. HIV and bone metabolism. Discov Med 2011; 11: 385-93.

[7] Weitzmann MN, Pacifici R. Estrogen deficiency and bone loss: An inflammatory tale. J Clin Invest 2006; 116: 1186-94.

[8] Weitzmann MN, Pacifici R. The role of T-lymphocytes in bone metabolism. Immunol Rev 2005; 208: 154-68.

[9] Vikulina $\mathrm{T}$, Fan X, Yamaguchi $\mathrm{M}$, et al. Alterations in the immuno-skeletal interface drive bone destruction in HIV-1 transgenic rats. Proc Natl Acad Sci USA 2010; 107: 13848-53.

[10] Titanji K, Vunnava A, Sheth AN, et al. Dysregulated B Cell Expression of RANKL and OPG Correlates with loss of bone mineral density in HIV infection. PLoS pathogens 2014; 10: e1004497.

[11] Makhluf HA, Mueller SM, Mizuno S, Glowacki J. Age-related decline in osteoprotegerin expression by human bone marrow cells cultured in three-dimensional collagen sponges. Biochem Biophys Res Commun 2000; 268: 669-72.

[12] Cao J, Venton L, Sakata T, Halloran BP. Expression of RANKL and OPG correlates with age-related bone loss in male C57BL/6 mice. J Bone Miner Res 2003; 18: 270-7.

[13] Cao JJ, Wronski TJ, Iwaniec U, et al. Aging increases stromal/osteoblastic cell-induced osteoclastogenesis and alters the osteoclast precursor pool in the mouse. J Bone Miner Res 2005; 20: 1659-68.

[14] Khosla S, Arrighi HM, Melton LJ, 3rd, et al. Correlates of osteoprotegerin levels in women and men. Osteoporos Int 2002; 13: 394-9.

[15] Indridason OS, Franzson L, Sigurdsson G. Serum osteoprotegerin and its relationship with bone mineral density and markers of bone turnover. Osteoporos Int 2005; 16: 417-23.

[16] Szulc P, Hofbauer LC, Heufelder AE, Roth S, Delmas PD. Osteoprotegerin serum levels in men: Correlation with age, estrogen, and testosterone status. J Clin Endocrinol Metab 2001; 86: 3162-5.

[17] Ikeda T, Utsuyama M, Hirokawa K. Expression profiles of receptor activator of nuclear factor kappaB ligand, receptor activator of nuclear factor kappaB, and osteoprotegerin messenger RNA in aged and ovariectomized rat bones. J Bone Miner Res 2001; 16: 1416-25.

[18] Weyand CM, Brandes JC, Schmidt D, Fulbright JW, Goronzy JJ. Functional properties of CD4+ CD28- T cells in the aging immune system. Mech Ageing Dev 1998; 102: 131-47.

[19] Goronzy JJ, Weyand CM. Aging, autoimmunity and arthritis: Tcell senescence and contraction of T-cell repertoire diversity catalysts of autoimmunity and chronic inflammation. Arthritis Res Ther 2003; 5: 225-34.

[20] Weksler ME. Changes in the B-cell repertoire with age. Vaccine 2000; 18: 1624-8.

[21] Salvioli S, Capri M, Valensin S, et al. Inflamm-aging, cytokines and aging: State of the art, new hypotheses on the role of mitochondria and new perspectives from systems biology. Curr Pharm Des 2006; 12: 3161-71.

[22] Toraldo G, Roggia C, Qian WP, Pacifici R, Weitzmann MN. IL-7 induces bone loss in vivo by induction of receptor activator of nuclear factor kappa B ligand and tumor necrosis factor alpha from T cells. Proc Natl Acad Sci USA 2003; 100: 125-30.

[23] Bouxsein ML, Boyd SK, Christiansen BA, Guldberg RE, Jepsen $\mathrm{KJ}$, Muller R. Guidelines for assessment of bone microstructure in rodents using micro-computed tomography. J Bone Miner Res 2010; 25: 1468-86

[24] Brodt MD, Ellis CB, Silva MJ. Growing C57Bl/6 mice increase whole bone mechanical properties by increasing geometric and material properties. J Bone Miner Res 1999; 14: 2159-66.

[25] Danilovich N, Sairam MR, Maysinger D. The menopausal mouse: A new neural paradigm of a distressing human condition. Neuroreport 2003; 14: 1617-22.

[26] Danilovich N, Babu PS, Xing W, Gerdes M, Krishnamurthy H, Sairam MR. Estrogen deficiency, obesity, and skeletal abnormalities in follicle-stimulating hormone receptor knockout (FORKO) female mice. Endocrinology 2000; 141: 4295-308.

[27] Sansoni P, Vescovini R, Fagnoni F, et al. The immune system in extreme longevity. Exp Gerontol 2008; 43(2): 61-5.

[28] Onal M, Xiong J, Chen X, et al. Receptor activator of nuclear factor kappaB ligand (RANKL) protein expression by $B$ lymphocytes contributes to ovariectomy-induced bone loss. J Biol Chem 2012; 287: 29851-60.

[29] Eghbali-Fatourechi G, Khosla S, Sanyal A, Boyle WJ, Lacey DL, Riggs BL. Role of RANK ligand in mediating increased bone resorption in early postmenopausal women. J Clin Invest 2003; 111: 1221-30.

[30] Kawai T, Matsuyama T, Hosokawa Y, et al. B and T lymphocytes are the primary sources of RANKL in the bone resorptive lesion of periodontal disease. Am J Pathol 2006; 169: 987-98.

[31] Choi Y, Woo KM, Ko SH, et al. Osteoclastogenesis is enhanced by activated B cells but suppressed by activated CD8(+) T cells. Eur J Immunol 2001; 31: 2179-88.

[32] Colucci S, Brunetti G, Cantatore F, et al. Lymphocytes and synovial fluid fibroblasts support osteoclastogenesis through RANKL, TNFalpha, and IL-7 in an in vitro model derived from human psoriatic arthritis. J Pathol 2007; 212: 47-55.

[33] Nanes MS. Tumor necrosis factor-alpha: molecular and cellular mechanisms in skeletal pathology. Gene 2003; 321: 1-15.

[34] Wahl EC, Aronson J, Liu L, et al. Restoration of regenerative osteoblastogenesis in aged mice: modulation of TNF. J Bone Miner Res 2010; 25: 114-23.

[35] Cenci S, Weitzmann MN, Roggia C, et al. Estrogen deficiency induces bone loss by enhancing T-cell production of TNF-alpha. J Clin Invest 2000; 106: 1229-37.

[36] Zhang YH, Heulsmann A, Tondravi MM, Mukherjee A, Abu-Amer Y. Tumor necrosis factor-alpha (TNF) stimulates RANKL-induced osteoclastogenesis via coupling of TNF type 1 receptor and RANK signaling pathways. J Biol Chem 2001; 276: 563-8.

[37] Fuller K, Murphy C, Kirstein B, Fox SW, Chambers TJ. TNF alpha potently activates osteoclasts, through a direct action independent of and strongly synergistic with RANKL. Endocrinology 2002; 143: 1108-18.

[38] Min H, Montecino-Rodriguez E, Dorshkind K. Effects of aging on the common lymphoid progenitor to pro-B cell transition. J Immunol 2006; 176: 1007-12.

[39] Colonna-Romano G, Bulati M, Aquino A, et al. B cells in the aged: CD27, CD5, and CD40 expression. Mech Ageing Dev 2003; 124 389-93. 
[40] Breitbart E, Wang X, Leka LS, Dallal GE, Meydani SN, Stollar BD. Altered memory B-cell homeostasis in human aging. J Gerontol 2002; 57: B304-11.

[41] Chong Y, Ikematsu H, Yamaji K, et al. CD27(+) (memory) B cell decrease and apoptosis-resistant CD27(-) (naive) B cell increase in aged humans: implications for age-related peripheral B cell developmental disturbances. Int Immunol 2005; 17: 383-90.
[42] Johnson SA, Cambier JC. Ageing, autoimmunity and arthritis: senescence of the B cell compartment - implications for humoral immunity. Arthritis Res Ther 2004; 6: 131-9.

[43] Hakim FT, Gress RE. Immunosenescence: deficits in adaptive immunity in the elderly. Tissue antigens 2007; 70: 179-89.

Received: July 15, 2014

Revised: November 11, 2014

Accepted: November 16, 2014

(C) Li et al.; Licensee Bentham Open.

This is an open access article licensed under the terms of the Creative Commons Attribution Non-Commercial License (http://creativecommons.org/licenses/bync/3.0/), which permits unrestricted, non-commercial use, distribution and reproduction in any medium, provided the work is properly cited. 